\title{
Cadastro Nacional de Estabelecimentos de Saúde como ferramenta de análise da descentralização do atendimento da tuberculose para a atenção básica
}

\section{Brazilian National Registry of Healthcare Establishments as a tool for the analysis of decentralization of tuberculosis treatment to basic care services}

\section{Registro Nacional de Establecimientos de} Salud como herramienta de análisis de la descentralización de la atención a la tuberculosis en la atención básica

\section{Resumo}

Os objetivos deste artigo foram validar um método de classificação dos serviços de saúde (atenção básica vs. outros níveis) e descrever a descentralização do atendimento da tuberculose (TB) para a atenção básica no Brasil no período de 2002 a 2016. Os serviços de saúde que notificaram e acompanharam pessoas com TB foram classificados como "atenção básica" ou "outros níveis", considerando-se o tipo de estabelecimento registrado no Cadastro Nacional de Estabelecimentos de Saúde (CNES). Foi estimada a concordância entre essa classificação e uma realizada em 2013 pelos programas estaduais e municipais de TB. Posteriormente, utilizando-se o CNES, calculou-se o percentual de pessoas com TB atendidas na atenção básica no período de 2002 a 2016. A concordância foi de 94,4\% e o indice kappa global foi 0,86. Houve um incremento relativo de $31,2 \%$ do atendimento de TB na atenção básica $(50,9 \%$ em 2002 para 66, 8\% em 2016). Todas as regiões apresentaram aumento desse percentual, exceto a Região Sul. A classificação baseada no CNES permitiu analisar a evolução da descentralização do atendimento da TB para a atenção básica no Brasil.

Tuberculose; Atenção Primária à Saúde; Sistemas de Informação em Saúde; Descentralização
Daniele Maria Pelissari 1,2

Marina Gasino Jacobs 1

Patricia Bartholomay ${ }^{1}$

Marli Souza Rocha 1

Draurio Barreira 3

Denise Arakaki-Sanchez 1

João Paulo Toledo 4

Fredi Alexander Diaz-Quijano 2

doi: 10.1590/0102-311X00173917
Correspondência

D. M. Pelissari

Programa Nacional de Controle da Tuberculose, Ministério da Saúde.

PO 700 - SRTVN 702, via W5 norte, 6o andar, Brasília, DF 70719-040, Brasil.

daniele.pelissari@gmail.com

1 Programa Nacional de Controle da Tuberculose, Ministério da Saúde, Brasília, Brasil.

2 Faculdade de Saúde Pública, Universidade de São Paulo, São Paulo, Brasil.

3 Unitaid, World Health Organization, Vernier, Switzerland. 4 Pan American Health Organization, Washington DC, U.S.A. 


\section{Introdução}

A atenção básica é considerada a principal porta de entrada da Rede de Atenção à Saúde no Brasil e o contato preferencial dos usuários com o Sistema Único de Saúde (SUS) 1. Em 2013, a cobertura populacional estimada da atenção básica no país foi de 69,3\% (DATASUS. http://tabnet.datasus.gov. br/cgi/deftohtm.exe?pacto/2013Mon/cnv/monitbr.def, acessado em 10/Out/2016).

Os serviços de atenção básica têm como uma de suas diretrizes a territorialização 1. Essa diretriz busca o desenvolvimento de ações setoriais e intersetoriais com foco em um território específico, de forma que atendam à necessidade de uma população cadastrada 1. Essa forma de organização, em conjunto com a descentralização e a maior vinculação entre pacientes e profissionais de saúde, favorecem a realização de atividades como a busca ativa de pessoas com tuberculose (TB), incluindo a investigação dos contatos e o seguimento clínico por meio do tratamento diretamente observado (TDO) 2,3,4,5.

Nesse sentido, desde 2004, o Ministério da Saúde, por meio do Programa Nacional de Controle da Tuberculose (PNCT), recomenda que a detecção e o acompanhamento das pessoas com TB pulmonar - forma clínica mais frequente da doença (87\% dos casos novos em 2015) - sejam realizados em serviços de atenção básica 5. São exceções a essa recomendação os casos de maior complexidade que devem ser assistidos em outros níveis (secundário e terciário), como é o caso da TB extrapulmonar, TB resistente aos fármacos antituberculose, coinfecção TB-HIV, falência de tratamento ou mudança de esquema por reações adversas 6 .

Apesar dessa recomendação, o sistema de informação em que são notificadas as pessoas com TB não classifica o nível de complexidade do serviço que os notifica e/ou acompanha. Dessa forma, não é possível quantificar o percentual de pessoas com TB assistidas pela atenção básica, bem como avaliar o acesso e a qualidade das ações para a detecção e seguimento do tratamento da TB nesses serviços.

Sobre os serviços ofertados na atenção básica, o estudo de maior abrangência no país, com 12.977 pessoas diagnosticadas com TB, utilizou uma classificação de estabelecimentos de saúde realizada por coordenadores de programas de TB 7 . Esse estudo constatou que 61,4\% das pessoas com TB pulmonar bacilífera foram diagnosticadas e tratadas por serviços de atenção básica. No entanto, esse trabalho limitou-se às capitais dos estados brasileiros e ao ano de 2013, não permitindo obter estimativas de todos os municípios, nem conhecer a evolução do processo de descentralização do cuidado da TB para a atenção básica 7 .

A validação de um método para identificar os estabelecimentos de atenção básica, com abrangência nacional, permitiria o monitoramento dos indicadores de TB nesses serviços para todos os municípios brasileiros. O Cadastro Nacional de Estabelecimentos de Saúde (CNES) pode ser uma alternativa para classificar os estabelecimentos. Esse cadastro caracteriza todos os serviços de saúde do SUS no Brasil, e pode ser utilizado para classificar aqueles que assistem pessoas com TB registradas no Sistema de Informação de Agravos de Notificação (SINAN).

Em consequência, o presente estudo propõe validar o CNES como uma ferramenta para um método de classificação dos serviços de saúde em atenção básica e outros níveis responsáveis pela assistência das pessoas com TB, com base nos casos diagnosticados em 2013 nas capitais brasileiras. Valendo-se dessa classificação, descrevemos a evolução da descentralização do atendimento de pessoas com TB para a atenção básica segundo macrorregião, Unidade Federativa (UF) e município, no período entre 2002 a 2016 no Brasil.

\section{Métodos}

\section{Desenho e população}

Adotou-se para este estudo a utilização do termo "atenção básica”, o qual, conforme a Política Nacional de Atenção Básica, é equivalente ao termo "atenção primária à saúde" 1 .

Este estudo foi desenvolvido em duas etapas. Na Etapa 1, foi realizada uma validação da classificação do nível de complexidade de serviços de saúde em atenção básica existente no CNES, estimando sua concordância com uma classificação realizada pelos coordenadores de programas de TB de municípios e estados, denominada Classificação de Estabelecimentos de Atenção à Tuberculose (CEAT). A 
CEAT considera os serviços de acordo com sua complexidade no que diz respeito exclusivamente ao atendimento das pessoas com TB. Nessa validação, foram incluídos os estabelecimentos de saúde que notificaram e atenderam pessoas com TB e os casos novos de TB elegíveis para serem acompanhados na atenção básica, de residentes nas capitais brasileiras em 2013.

Na Etapa 2, utilizando-se a classificação do CNES, descrevemos a descentralização do atendimento às pessoas com TB elegíveis para acompanhamento na atenção básica, diagnosticadas no período de 2002 a 2016 no Brasil.

O Ministério da Saúde recomenda que casos de TB de baixa complexidade sejam diagnosticados e acompanhados pela atenção básica, e aqueles que demandam diagnósticos e conduta terapêutica de elevada complexidade, por unidades de referência para TB 5 . Nesse sentido, a população incluída neste estudo foram os pacientes com TB com indicação de acompanhamento por serviços de atenção básica, ou seja, casos novos de TB, da forma clínica pulmonar e que não apresentavam registro de nenhuma das seguintes condições: TB resistente aos fármacos antituberculose, coinfecção TB-HIV, falência de tratamento ou mudança de esquema por reações adversas.

\section{Fonte de dados}

Os casos de TB e os estabelecimentos de saúde que os assistiram (notificaram e acompanharam os pacientes) foram extraídos do SINAN no ano de 2013 para a Etapa 1, e 2002 a 2016 para a Etapa 2. A seguir estão descritas as duas fontes de dados usadas como parâmetros para as classificações dos estabelecimentos de saúde segundo o nível de atenção:

(a) CNES: tem abrangência nacional e contempla todos os serviços de saúde do SUS cadastrados pelas UFs de 2007 a 2016, ativos ou não. O tipo de unidade do serviço de saúde é definido pelo gestor do estabelecimento, com atualização mensal. No presente estudo, adotou-se o critério do Departamento de Atenção Básica (DAB) do Ministério da Saúde, que considera os seguintes tipos como serviços de atenção básica: posto de saúde, centro de saúde/unidade básica, unidade mista, unidade móvel fluvial e terrestre. Os demais estabelecimentos de saúde foram classificados como "outros serviços". Uma segunda análise foi realizada considerando-se as unidades mistas como "outros serviços", baseandose no conceito de que alguns destes serviços poderiam ser unidades de referência secundária para o atendimento de pessoas com TB.

(b) CEAT: abrange as capitais brasileiras, está disponível para o ano de 2013 e contempla os serviços de saúde que assistiram os casos de TB residentes nestas cidades, tendo já sido utilizada em estudo prévio 7. A classificação foi realizada pelos Programas Estaduais de Controle da Tuberculose (PECT) e Programas Municipais de Controle da Tuberculose (PMCT). Os estabelecimentos foram classificação em: (i) atenção básica (nível de menor densidade tecnológica para atendimento de pessoas com TB; p.ex.: unidade básica de saúde e posto de saúde); (ii) atenção secundária (referência secundária para o tratamento de TB, com densidade tecnológica intermediária; p.ex.: Serviço de Atendimento Especializado e Unidade de Pronto Atendimento); (iii) atenção terciária (referência terciária para o tratamento de TB, com maior densidade tecnológica; p.ex.: hospitais); e (iv) outros (vigilância epidemiológica, laboratórios e serviços privados). Neste estudo os serviços classificados como atenção secundária, terciária e outros compuseram a categoria "outros serviços" 7.

\section{- Etapa 1 - concordância da classificação dos estabelecimentos de saúde em serviços de atenção básica}

Cada estabelecimento de saúde cadastrado no CNES tem um código único que é registrado em todas as notificações de TB do SINAN pelas variáveis "unidade de notificação" e "unidade de atendimento atual". Inicialmente, extraímos do SINAN esses códigos de estabelecimentos que assistiram pessoas com TB residentes nas capitais, diagnosticadas em 2013. Em seguida, incluímos a essa lista de estabelecimentos a classificação do nível de atenção pelos critérios do CNES e da CEAT.

A concordância entre as classificações do CNES e da CEAT foi avaliada pelo índice kappa global e desagregada (por capital), com os seus respectivos intervalos de 95\% de confiança (IC95\%). Valores do índice kappa acima de 0,80 foram considerados como concordância quase perfeita, entre 0,61 e 0,80 concordância substancial, entre 0,41 e 0,60 como moderada, e abaixo de 0,40, baixa concordância 8 . 
Adicionalmente, os casos novos de TB elegíveis para serem acompanhados na atenção básica receberam as classificações CNES e CEAT com base nos estabelecimentos de saúde que realizaram o seu acompanhamento ("unidade de atendimento atual"). A proporção desses casos que foi atendida na atenção básica segundo essas duas classificações foi comparada utilizando-se o teste McNemar para cada capital 9 . Devido às 27 comparações, e considerando-se a correção de Bonferroni, foi estabelecido um nível de significância de 0,002 10 .

\section{- Etapa 2 - descentralização do atendimento de pessoas com TB elegíveis para serem acompanhadas na atenção básica}

Nesta análise foram considerados os casos de TB elegíveis para acompanhamento na atenção básica, diagnosticados no período de 2002 a 2016 em todos os municípios registrados no SINAN. Esses pacientes foram classificados segundo seus respectivos estabelecimentos de atendimento: "atenção básica" ou "outros serviços" pelo critério do CNES. A classificação do estabelecimento de saúde pode mudar ao longo do tempo pela incorporação ou desincorporação de tecnologias, procedimentos e especialidades pelos estabelecimentos. No entanto, neste estudo foi considerada a última classificação do CNES registrada durante o período da pesquisa, devido à ausência de ferramentas para a extração de dados retrospectivos. Em seguida, calculamos o percentual de casos de TB elegíveis que foram atendidos por serviços de atenção básica.

Esse indicador foi apresentado graficamente em uma série histórica para o país e por macrorregião. No que se refere às UFs, estimamos os coeficientes de regressão de Poisson e os respectivos IC95\% para avaliar a tendência temporal (2002 a 2016) da proporção de pessoas com TB elegíveis para acompanhamento na atenção básica, atendidas neste nível de atenção para cada UF. Adicionalmente, a evolução desse indicador por municípios foi apresentada por meio de mapas indicadores, tomando-se como exemplos os anos 2002, 2009 e 2016.

Todas as análises foram realizadas no Stata, versão 12 (https://www.stata.com) e os mapas foram feitos no QGIS-Geographic Information System, versão 2.18.15 (https://www.qgis.org/en/site/). O presente estudo foi aprovado pela Comissão Nacional de Ética em Pesquisa (parecer no 1.810.520).

\section{Resultados}

\section{Concordância da classificação dos estabelecimentos de saúde em serviços de atenção básica}

Em 2013, foram registrados 19.979 casos novos de TB elegíveis para o atendimento na atenção básica dos residentes nas capitais, dos quais 19.419 (97,2\%) foram atendidos em estabelecimentos de saúde classificados segundo os critérios do CNES e da CEAT. Esses casos foram notificados ou atendidos por 4.983 estabelecimentos de saúde.

O kappa global dos estabelecimentos de saúde não sofreu alteração quando comparado à classificação que considerou unidade mista como "atenção básica", com aquela que a classificou como "outros serviços”. No entanto, três municípios apresentaram melhoria do índice kappa pela classificação de unidade mista como “outros serviços". São Luís passou de 0,82 para 0,97; Natal de 0,74 para 0,80; e Teresina de 0,90 para 1,00. Nesse sentido, optou-se por utilizar a classificação do CNES que considerou as unidades mistas como "outros serviços" para o desenvolvimento do restante das análises.

Considerando-se essa classificação do CNES (unidades mistas classificadas como "outros serviços”), 3.452 dos estabelecimentos de saúde que notificaram e/ou atenderam casos de TB foram classificados como atenção básica. Por outra parte, segundo a classificação da CEAT o número de estabelecimentos desse nível de atenção seria de 3.561. A concordância nacional dessas duas classificações foi de $94,4 \%$, sendo que todos os municípios apresentaram valores acima de 85\% (Figura 1a).

O índice kappa global foi de 0,86 (IC95\%: 0,84; 0,87). Nas capitais Rio Branco, Maceió, Salvador e Brasília, os índices kappa indicaram concordância substancial $(0,61$ a 0,80). Nas demais, a concordância foi quase perfeita (acima de 0,80), ou perfeita, como é o caso de: Vitória, Aracaju, Campo Grande, Teresina e Curitiba (Figura 1b). 


\section{Figura 1}

Percentual de concordância, índice kappa e intervalos de 95\% de confiança (IC95\%) entre os critérios do CNES * e da CEAT ** para as classificações dos estabelecimentos de saúde que notificaram/atenderam pessoas com tuberculose segundo o nível de complexidade de atenção. Capitais, Brasil, 2013.

1a) Concordância (\%)

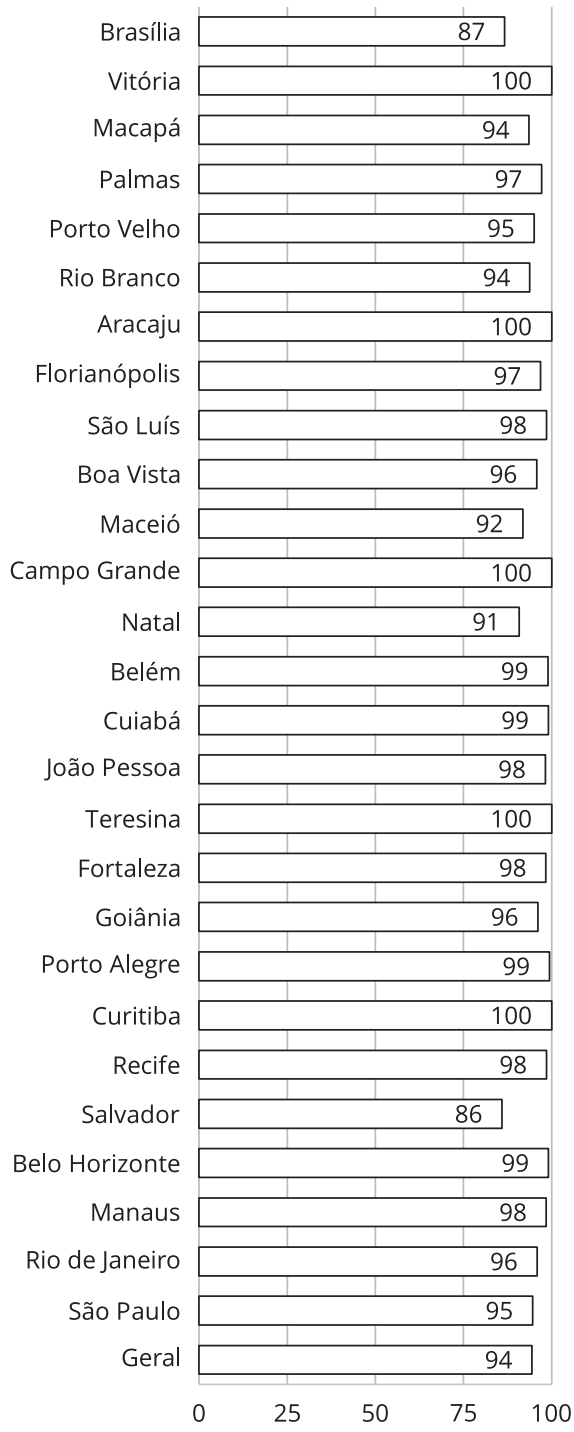

1b) Índice kappa e IC95\%

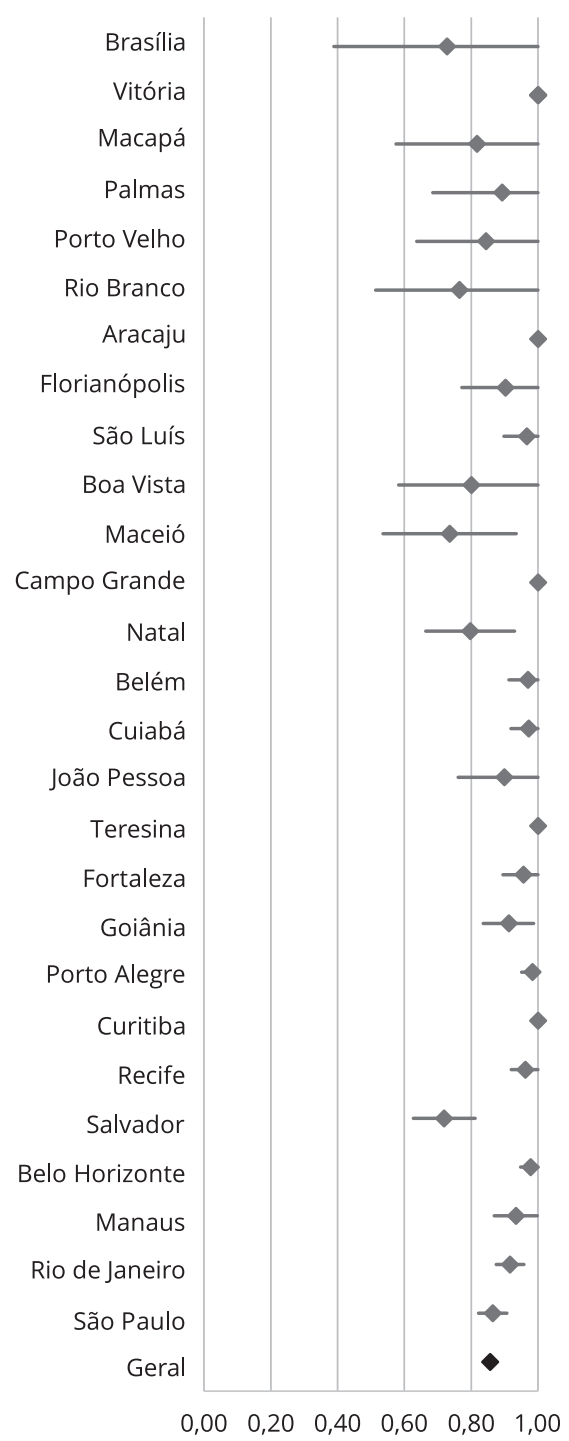

CEAT: Classificação de Estabelecimentos de Atenção à Tuberculose; CNES: Cadastro Nacional de Estabelecimentos de Saúde.

* CNES: atenção básica (posto de saúde, centro de saúde/unidade básica, unidade móvel fluvial e terrestre) vs. outros serviços (incluindo unidades mistas);

** CEAT: atenção básica vs. outros serviços.

Quanto ao nível de atenção dos estabelecimentos de saúde que realizaram o acompanhamento dos casos de TB elegíveis para serem acompanhados na atenção básica, as capitais Manaus, Belém, São Luís, Maceió e São Paulo apresentaram diferença estatisticamente significativa $(\mathrm{p}<0,002)$ nas proporções segundo os dois critérios de classificação dos estabelecimentos (CNES e CEAT). A diferença 
relativa entre as proporções obtidas com as duas classificações foi superior a 10\% nos municípios de Manaus (11,1\%), São Luís $(19,6 \%)$ e Maceió $(-27,4 \%)$. No entanto, no que se refere ao valor total das capitais do Brasil, a diferença relativa foi de $-1,2 \%$ (Tabela 1).

\section{Descentralização do atendimento de pessoas com TB elegíveis para serem acompanhadas na atenção básica}

Considerando-se o resultado obtido ao comparar a classificação do CNES com a CEAT, a classificação baseada no CNES foi usada para avaliar as tendências de descentralização do atendimento da TB para a atenção básica em todo o país. Assim, no Brasil, o percentual de casos de TB atendidos por serviços de atenção básica passou de 50,9\% em 2002 para 66,8\% em 2016, perfazendo um incremento relativo de 31,2\% do atendimento de TB na atenção básica. Com exceção da Região Sul, todas as demais regiões apresentaram aumento desse percentual, com destaque para a Região Norte, que

Tabela 1

Proporção de casos novos de tuberculose (TB) *, atendidos na atenção básica segundo critérios CEAT e CNES. Capitais, Brasil.

\begin{tabular}{|c|c|c|c|c|c|}
\hline \multirow[t]{2}{*}{ Município } & \multirow{2}{*}{$\begin{array}{l}\text { Casos elegíveis para } \\
\text { serem acompanhados na } \\
\text { atenção básica }\end{array}$} & \multicolumn{2}{|c|}{$\begin{array}{l}\text { Casos de TB cujos estabelecimentos foram } \\
\text { classificados como atenção básica [n (\%)] }\end{array}$} & \multirow[t]{2}{*}{ Diferença absoluta } & \multirow[t]{2}{*}{ Diferença relativa } \\
\hline & & CEAT & CNES & & \\
\hline Porto Velho & 232 & $180(77,6)$ & $180(77,6)$ & 0,0 & 0,0 \\
\hline Rio Branco & 183 & $154(84,2)$ & $148(80,9)$ & $-3,3$ & $-3,9$ \\
\hline Manaus & 1.176 & $646(54,9)$ & $718(61,1)$ & 6,1 & $11,1 * *$ \\
\hline Boa Vista & 20 & $15(75,0)$ & $15(75,0)$ & 0,0 & 0,0 \\
\hline Belém & 1.103 & $997(90,4)$ & $938(85)$ & $-5,3$ & $-5,9 * *$ \\
\hline Macapá & 87 & $43(49,4)$ & $41(47,1)$ & $-2,3$ & $-4,7$ \\
\hline Palmas & 31 & $28(90,3)$ & $28(90,3)$ & 0,0 & 0,0 \\
\hline São Luís & 418 & $102(24,4)$ & $122(29,2)$ & 4,8 & $19,6 * *$ \\
\hline Teresina & 339 & $256(75,5)$ & $256(75,5)$ & 0,0 & 0,0 \\
\hline Fortaleza & 1.138 & $1.004(88,2)$ & $1.006(88,4)$ & 0,2 & 0,2 \\
\hline Natal & 332 & $250(75,3)$ & $262(78,9)$ & 3,6 & 4,8 \\
\hline João Pessoa & 266 & $66(24,8)$ & $66(24,8)$ & 0,0 & 0,0 \\
\hline Recife & 1.192 & $768(64,4)$ & $769(64,5)$ & 0,1 & 0,1 \\
\hline Maceió & 397 & $369(92,9)$ & $268(67,5)$ & $-25,4$ & $-27,4 * *$ \\
\hline Aracaju & 155 & $107(69,0)$ & $107(69,0)$ & 0,0 & 0,0 \\
\hline Salvador & 1.396 & $854(61,2)$ & $853(61,1)$ & $-0,1$ & $-0,1$ \\
\hline Belo Horizonte & 424 & $311(73,3)$ & $330(77,8)$ & 4,5 & 6,1 \\
\hline Vitória & 123 & $105(85,4)$ & $105(85,4)$ & 0,0 & 0,0 \\
\hline Rio de Janeiro & 4.114 & $3.406(82,8)$ & $3.386(82,3)$ & $-0,5$ & $-0,6$ \\
\hline São Paulo & 3.954 & $3.317(83,9)$ & $3.199(80,9)$ & $-3,0$ & $-3,6 * *$ \\
\hline Curitiba & 249 & $214(85,9)$ & $220(88,4)$ & 2,4 & 2,8 \\
\hline Florianópolis & 144 & $109(75,7)$ & $108(75,0)$ & $-0,7$ & $-0,9$ \\
\hline Porto Alegre & 811 & $201(24,8)$ & $205(25,3)$ & 0,5 & 2,0 \\
\hline Campo Grande & 137 & $88(64,2)$ & $88(64,2)$ & 0,0 & 0,0 \\
\hline Cuiabá & 638 & $570(89,3)$ & $569(89,2)$ & $-0,2$ & $-0,2$ \\
\hline Goiânia & 151 & $69(45,7)$ & $67(44,4)$ & $-1,3$ & $-2,9$ \\
\hline Brasília & 209 & $118(56,5)$ & $118(56,5)$ & 0,0 & 0,0 \\
\hline Capitais & 19.419 & $14.347(73,9)$ & $14.172(73,0)$ & $-0,9$ & $-1,2 * *$ \\
\hline
\end{tabular}

CEAT: Classificação de Estabelecimentos de Atenção à Tuberculose; CNES: Cadastro Nacional de Estabelecimentos de Saúde.

* Elegíveis para serem acompanhados na atenção básica;

** Valor de $p<0,002$ (teste McNemar). 
passou de 37,9\% (2002) para 80,1\% (2016). A região Centro-oeste passou de 48,3\% para 67,8\% de 2002 a 2012 e, em seguida, apresentou uma redução para 56\% até 2016 (Figura 2).

A análise de tendência por UF indicou que o percentual de pessoas com TB atendidas na atenção básica foi estável nos estados de Sergipe, Mato Grosso, Mato Grosso do Sul e Santa Catarina, e no Distrito Federal, no período de 2002 a 2016 ( $p>0,05$ ). No Rio Grande do Sul, esse percentual reduziu (coeficiente de regressão -0,29; IC95\%: -0,32; -0,26) e em todos os demais estados apresentou tendência positiva $(\mathrm{p}<0,05)$ (Tabela 2$)$.

Na representação dos municípios brasileiros, observou-se que o processo de descentralização da assistência das pessoas com TB para a atenção básica foi maior nas regiões Norte e Nordeste quando comparados os mapas dos anos 2002, 2009 e 2016 (Figura 3). O percentual de municípios onde mais de $66 \%$ dos pacientes elegíveis para serem acompanhados na atenção básica foram atendidos nestes serviços passou de 54,9\% (2.138/3.891) em 2002 para 62,7\% em 2009 (2.343/3.736) e 66,1\% em 2016 $(2.386 / 3.609)$.

\section{Discussão}

Nosso estudo avaliou o CNES como uma estratégia para a classificação de estabelecimentos de saúde segundo nível de atenção, com um recorte específico para o cuidado de pessoas com TB, diferenciando a atenção básica dos outros níveis de atenção. Nesta avaliação, o CNES demonstrou ser uma ferramenta consistente para monitorar o percentual de pessoas com TB atendidas na atenção básica, por apresentar um elevado índice kappa e uma diferença relativa de apenas 1,2\% na estimação da proporção de casos atendidos nestes serviços, em comparação com a classificação CEAT.

Os resultados encontrados com o relacionamento dessa classificação aos casos de TB demonstraram o aumento da descentralização do cuidado de pessoas com TB para a atenção básica no Brasil. Considerando-se todos os níveis de agregação analisados (macrorregião, UF e município), identificamos que esse processo foi mais intenso nos municípios e estados das regiões Sudeste, Norte e Nordeste. O mesmo não foi observado na Região Sul, especificamente no Estado do Rio Grande do Sul que apresentou tendência negativa, apesar de esforços no sentido de fortalecer e descentralizar

\section{Figura 2}

Percentual de casos novos de tuberculose * atendidos na atenção básica. Brasil e macrorregiões, 2002 a 2016.

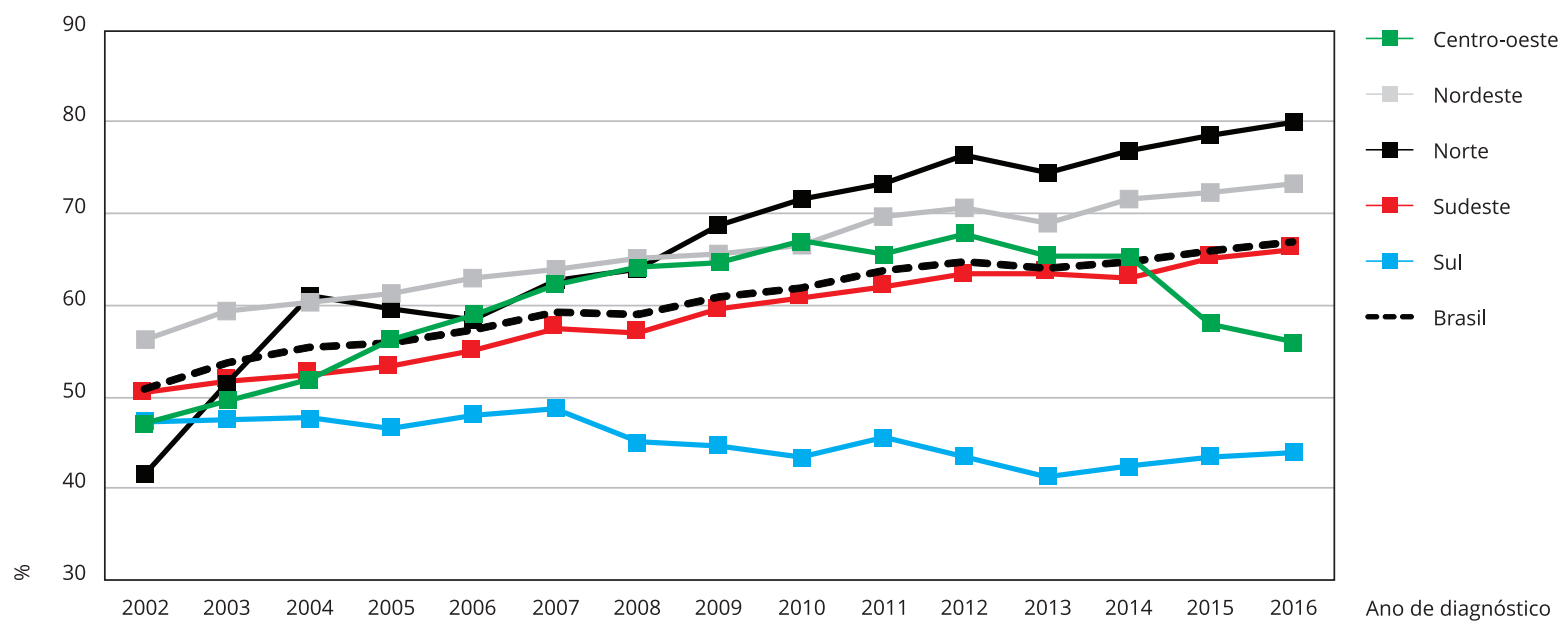

* Elegíveis para serem acompanhados na atenção básica. 
Tabela 2

Casos novos de tuberculose (TB) * atendidos na atenção básica e coeficientes de regressão de Poisson da tendência. Brasil, 2002,2009 e 2016.

\begin{tabular}{|c|c|c|c|c|}
\hline \multirow[t]{2}{*}{ Unidade Federativa } & \multicolumn{3}{|c|}{ Casos novos de TB na atenção básica [n (\%)] } & \multirow{2}{*}{$\begin{array}{l}\text { Coeficiente de regressão ** } \\
\text { (IC95\%) }\end{array}$} \\
\hline & 2002 & 2009 & 2016 & \\
\hline \multicolumn{5}{|l|}{ Região Norte } \\
\hline Acre & $49(19,6)$ & $176(72,1)$ & $261(79,6)$ & $0,53(0,44 ; 0,62) * * *$ \\
\hline Amapá & $50(24,0)$ & $77(39,3)$ & $116(62,7)$ & $0,62(0,49 ; 0,75) * * *$ \\
\hline Amazonas & $127(7,6)$ & $1.005(59,4)$ & $1.321(69,4)$ & $0,60(0,56 ; 0,64) * \star \star$ \\
\hline Pará & $1.838(65,3)$ & $2.252(79,8)$ & $2.559(88,0)$ & $0,21(0,19 ; 0,24) * * *$ \\
\hline Rondônia & $147(31,9)$ & $202(43,1)$ & $363(81,0)$ & $0,67(0,59 ; 0,75) * \star \star$ \\
\hline Roraima & $45(44,6)$ & $49(70,0)$ & $73(82,0)$ & $0,26(0,11 ; 0,41) * * *$ \\
\hline Tocantins & $139(59,9)$ & $113(81,3)$ & $106(79,7)$ & $0,32(0,22 ; 0,43) * * *$ \\
\hline \multicolumn{5}{|l|}{ Região Nordeste } \\
\hline Alagoas & $672(69,4)$ & $587(62,4)$ & $510(70,2)$ & $0,08(0,03 ; 0,13) * * \star$ \\
\hline Bahia & $2.999(59,4)$ & $2.851(59,9)$ & $2.347(70,1)$ & $0,12(0,10 ; 0,14) * * *$ \\
\hline Ceará & $2.257(72,1)$ & $2.808(87,4)$ & $2.154(86,2)$ & $0,11(0,09 ; 0,14) * * *$ \\
\hline Maranhão & $1.401(58,5)$ & $1.060(58,5)$ & $1.031(65,0)$ & $0,04(0,00 ; 070) * * *$ \\
\hline Paraíba & $364(39,9)$ & $376(44,7)$ & $476(58,8)$ & $0,09(0,04 ; 0,15) * \star *$ \\
\hline Pernambuco & $922(28,8)$ & $1.751(56,3)$ & $2.271(70,0)$ & $0,49(0,46 ; 0,52) * \star \star$ \\
\hline Piauí & $471(50,4)$ & $503(71,1)$ & $405(81,7)$ & $0,34(0,28 ; 0,39) * * *$ \\
\hline Rio Grande do Norte & $653(71,2)$ & $624(79,9)$ & $633(82,4)$ & $0,17(0,12 ; 0,22) * \star \star$ \\
\hline Sergipe & $315(84,5)$ & $353(74,3)$ & $429(79,3)$ & $0,01(-0,05 ; 0,07)$ \\
\hline \multicolumn{5}{|l|}{ Região Sudeste } \\
\hline Espírito Santo & $438(42,4)$ & $462(47,8)$ & $451(56,6)$ & $0,23(0,18 ; 0,29) * \star \star$ \\
\hline Minas Gerais & $1.827(53,0)$ & $1.708(56,1)$ & $1.380(57,7)$ & $0,05(0,02 ; 0,08) * * *$ \\
\hline Rio de Janeiro & $5.456(53,7)$ & $4.846(55,5)$ & $4.731(58,7)$ & $0,09(0,08 ; 0,11) * \star \star$ \\
\hline São Paulo & $5.285(47,4)$ & $7.031(64,9)$ & $9.368(73,0)$ & $0,28(0,27 ; 0,29) * \star \star$ \\
\hline \multicolumn{5}{|l|}{ Região Centro-oeste } \\
\hline Distrito Federal & $76(34,9)$ & $106(60,2)$ & $93(42,1)$ & $0,08(-0,13 ; 0,18)$ \\
\hline Goiás & $229(33,2)$ & $323(48,6)$ & $381(54,9)$ & $0,44(0,38 ; 0,51) * * *$ \\
\hline Mato Grosso & $502(58,8)$ & $631(80,2)$ & $535(61,0)$ & $0,04(-0,11 ; 0,09)$ \\
\hline Mato Grosso do Sul & $286(51,1)$ & $421(63,7)$ & $380(55,2)$ & $0,02(-0,14 ; 0,09)$ \\
\hline \multicolumn{5}{|l|}{ Região Sul } \\
\hline Paraná & $1.079(53,2)$ & $1.085(62,5)$ & $879(58,4)$ & $0,14(0,10 ; 0,17) * * *$ \\
\hline Rio Grande do Sul & $1.451(49,1)$ & $1.215(36,3)$ & $1.116(39,3)$ & $-0,29(-0,32 ;-0,26) * \star *$ \\
\hline Santa Catarina & $288(29,9)$ & $436(41,8)$ & $437(36,9)$ & $0,06(-0,11 ; 0,12)$ \\
\hline Brasil & $29.617(50,9)$ & $33.058(60,9)$ & $34.814(66,8)$ & $0,18(0,17 ; 0,18) * \star \star$ \\
\hline
\end{tabular}

* Elegíveis para serem acompanhados na atenção básica;

** Coeficientes de regressão (modelo de Poisson) estimados para cada acréscimo de 10 anos da variável ano de diagnóstico;

$\star * * \mathrm{p}<0,05$.

o atendimento de pessoas com TB para a atenção básica 11. Na Região Centro-oeste, as causas da queda nos últimos dois anos do atendimento de pessoas com TB na atenção básica são desconhecidas, baseadas nos dados deste estudo e na literatura científica. Possíveis causas a serem investigadas seriam problemas relacionados à qualidade da informação ou tendência centralizadora da organização do fluxo dos pacientes na rede de atenção dessa região.

Os resultados obtidos para as regiões Norte e Nordeste acompanham o processo histórico da Estratégia Saúde da Família - ESF (anteriormente conhecida como Programa Saúde da Família - PSF) que expandiu a atenção básica, alcançando as regiões mais pobres do país, pequenas cidades e bairros periféricos nas áreas metropolitanas 12 


\section{Figura 3}

Percentual de casos novos de tuberculose (TB) * atendidos na atenção básica. Municípios, Brasil, 2002,2009 e 2016.

3a) 2002

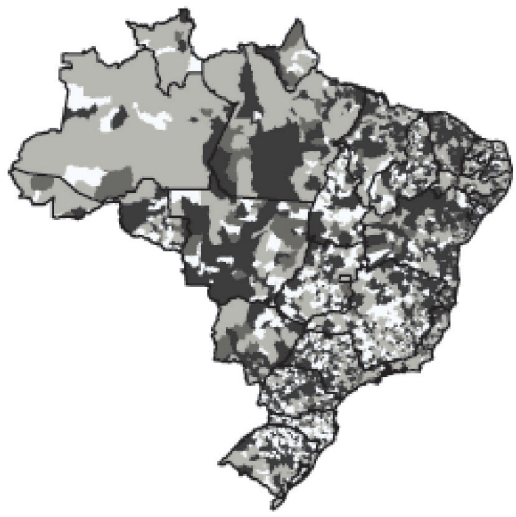

3b) 2009

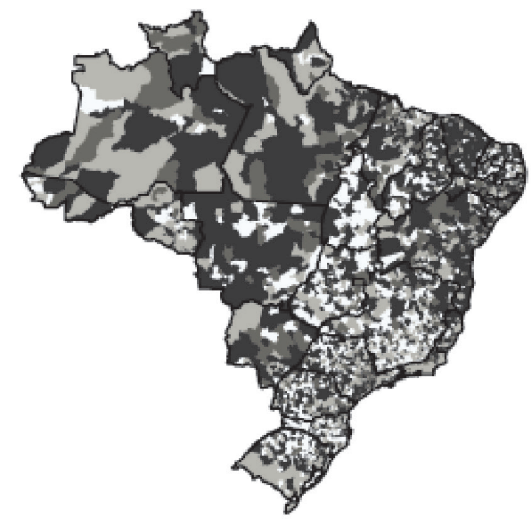

3c) 2016

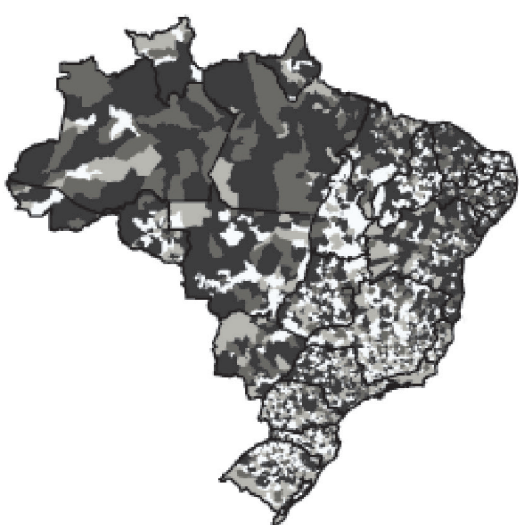

Casos de TB na atenção básica (\%)

$0 \nmid 33$

$33 \dashv 66$

$66-100$

Sem casos

* Elegíveis para serem acompanhados na atenção básica.

O percentual de municípios onde pelo menos $66 \%$ das pessoas com TB foram atendidas na atenção básica apresentou um maior incremento entre os anos 2002 e 2009 (que foi de 7,8\%) do que entre 2009 e 2016 (incremento de 3,4\%). Isso pode representar etapas distintas do processo histórico de descentralização do atendimento de pessoas com TB para a atenção básica.

Tendo em vista que o Brasil é marcado por disparidades sociais que o dividem em dois cenários socioeconômicos, agrupando as regiões Norte e Nordeste no menos favorecido e as demais no mais favorecido 13,14, esperava-se que a descentralização para a atenção básica fosse distribuída de forma heterogênea entre os municípios brasileiros por macrorregiões, o que não foi constatado em 2016. Nesse processo, enfatiza-se, após décadas de modelo assistencial centralizado no hospital, o papel indutor do governo central para a transferência aos municípios da responsabilidade com a atenção básica. Para esse fim foram desenvolvidas ações específicas como é o caso da ESF 15,16, que contemplou explicitamente o cuidado da TB na atenção básica 17,18,19. Além disso, outro impulsionador desse movimento foi a incorporação dessa pauta na agenda do PNCT 20, e em consequência dos programas locais de TB.

No que se refere à realização do atendimento da TB na atenção básica, ainda incipiente em algumas UFs, como é o caso das regiões Sul e Centro-oeste, estudos brasileiros revelam diversas barreiras neste nível de menor densidade tecnológica que poderiam afetar as ações de TB nestes serviços. Dentre elas, destacam-se: recursos humanos insuficientes 21,22; o fato de que os profissionais não acompanham necessariamente as mudanças nas condições e práticas nos serviços 23; e as características distintas dos sistemas de saúde implantados nos municípios, o que levou a uma extrema diversidade de grau de inserção da ESF na atenção básica 22. Em um estudo realizado nos estados do Sudeste, a debilidade quantitativa e qualitativa de recursos humanos e a visão centralizada e fragmentada da 
organização dessas ações no sistema de saúde foram identificadas como barreiras para o cuidado da TB na atenção básica 24 .

Entre as limitações deste estudo está a qualidade dos registros das variáveis no SINAN, que classificam as pessoas com TB como elegíveis para serem acompanhados na atenção básica. Além disso, a classificação dos tipos de estabelecimentos de saúde no CNES, que pode variar para cada macrorregião, UF e município. Nesse sentido, espera-se algum viés de classificação, o que pode interferir no indicador sobre a descentralização analisado no presente estudo.

Outra limitação sobre a classificação dos casos de TB elegíveis para serem acompanhados na atenção básica é que doenças ou condições, além daquelas informadas na ficha de notificação/investigação do SINAN, como, por exemplo, outras imunossupressões que não o HIV e pacientes renais crônicos 5 , podem requerer o atendimento em instituições de maior nível de complexidade. Logo, esses pacientes foram classificados como elegíveis para o acompanhamento na atenção básica, o que causaria uma subestimação do indicador, tendo em vista o inflacionamento do denominador.

Adicionalmente, a base de dados do CNES utilizada neste estudo considerou a última classificação realizada pelo gestor no período de 2007 a 2016. No entanto, um serviço de saúde inicialmente classificado como unidade básica, pela incorporação de uma determinada especialidade, poderia se tornar uma referência secundária posteriormente. Em períodos de crescimento econômico, como foi o caso do Brasil até o ano de 2014, espera-se com maior frequência que os serviços de atenção básica aumentassem o seu nível de complexidade ao longo do tempo, pela incorporação de tecnologia e especialidades. Isso pode ter subestimado o indicador analisado, principalmente nos anos iniciais do período estudado. Por fim, anos que antecedem 2007 podem ter maior limitação, uma vez que a base de dados do CNES passou a ser consolidada a partir de 2007. Apesar da impossibilidade de resgatar as informações retrospectivas, prospectivamente é possível monitorar esses dados na medida em que sejam solicitados como parte das rotinas dos programas de TB.

Apesar dessas limitações, este estudo traz de forma inédita uma descrição do processo histórico da descentralização do cuidado da TB para a atenção básica no Brasil. Isso fornece um panorama que pode orientar a tomada de decisões em políticas públicas, em particular aquelas relativas ao fortalecimento desses serviços de saúde. O fortalecimento da atenção básica já foi associado inversamente à mortalidade por diversas doenças em um estudo que analisou dados secundários de 18 países, mesmo quando ajustado por determinantes socioeconômicos e de cobertura de serviços de saúde 25 . No caso da TB, no Brasil a busca ativa de casos e a vigilância de contatos realizadas por equipes da atenção básica foram associadas ao aumento da detecção da doença nos territórios 26 . Nesse mesmo estudo, a cobertura da atenção básica esteve inversamente associada à taxa de incidência da TB, o que poderia representar a contribuição da atenção básica sobre o controle da transmissão, provavelmente, com base no diagnóstico oportuno e no tratamento precoce.

Estudos futuros podem utilizar o método que validamos para explorar indicadores de qualidade da prestação dos serviços de TB pela atenção básica, uma vez que se torna possível o cálculo e o monitoramento de indicadores operacionais específicos para estes serviços, tais como o percentual de realização de testes para o HIV e o percentual de cura nas pessoas com TB. Adicionalmente, com as devidas adaptações, o método que validamos poderá ser utilizado para o monitoramento dos serviços prestados na atenção básica de outras doenças e agravos.

Em síntese, nosso estudo apresenta a validação de um método para classificar estabelecimentos de saúde em atenção básica que acompanham pessoas com TB básica por intermédio do CNES. Esse método utiliza bases de dados que estão disponíveis, o que facilita a sua operacionalização e possibilita o monitoramento do processo de descentralização do cuidado dessas pessoas para a atenção básica nos municípios brasileiros. Por fim, os resultados do presente estudo sugerem que houve um aumento dessa descentralização, e que, no entanto, esse processo não foi homogêneo para todo o país. 


\section{Colaboradores}

D. M. Pelissari, M. G. Jacobs e P. Bartholomay conceberam o projeto. D. M. Pelissari e F. A. Diaz-Quijano planejaram e realizaram as análises e as ilustrações. D. M. Pelissari fez a redação da primeira versão. Todos os autores interpretaram os dados, realizaram a revisão crítica relevante do conteúdo intelectual, aprovaram a versão final e são responsáveis por todos os aspectos do trabalho na garantia da exatidão e integridade de qualquer parte da obra.

\section{Agradecimentos}

Agradecemos ao Departamento de Atenção Básica, Secretaria de Atenção à Saúde, Ministério da Saúde, pelos esclarecimentos quanto à base de dados do Cadastro Nacional de Estabelecimentos de Saúde e classificação dos estabelecimentos.

\section{Referências}

1. Ministério da Saúde. Portaria no 2.436, de 21 de setembro de 2017. Aprova a Política Nacional de Atenção Básica, estabelecendo a revisão de diretrizes para a organização da Atenção Básica, no âmbito do Sistema Único de Saúde (SUS). Diário Oficial da União 2017; 22 set.

2. Cardozo-Gonzales RI, Palha PF, Harter J, Alarcon E, Lima LM, Tomberg JO. Avaliação das ações de detecção de casos de tuberculose na atenção primária. Rev Eletrônica Enferm 2016; 17(4). https://www.revistas.ufg.br/fen/ article/view/32846/20697.

3. Sulis G, Centis R, Sotgiu G, D’Ambrosio L, Pontali E, Spanevello A, et al. Recent developments in the diagnosis and management of tuberculosis. NPJ Prim Care Respir Med 2016; 26:16078

4. Eang MT, Satha P, Yadav RP, Morishita F, Nishikiori N, van-Maaren $\mathrm{P}$, et al. Early detection of tuberculosis through community-based active case finding in Cambodia. BMC Public Health 2012; 12:469.

5. Secretaria de Vigilância em Saúde, Ministério da Saúde. Manual de recomendações para o controle da tuberculose no Brasil. Brasília: Ministério da Saúde; 2011. (Séria A. Normas e Manuais Técnicos).

6. Figueiredo TMRM, Villa TCS, Scatena LM, Gonzales RIC, Ruffino-Netto A, Nogueira JA, et al. Desempenho da atenção básica no controle da tuberculose. Rev Saúde Pública 2009; 43:825-31.

7. Bartholomay P, Pelissari DM, de Araujo WN, Yadon ZE, Heldal E. Quality of tuberculosis care at different levels of health care in Brazil in 2013. Rev Panam Salud Pública 2016; 39: 3-11.

8. Landis JR, Koch GG. The measurement of observer agreement for categorical data. Biometrics 1977; 33:159-74.

9. Westfall PH, Troendle JF, Pennello G. Multiple McNemar tests. Biometrics 2010; 66:1185-91.

10. Armstrong RA. When to use the Bonferroni correction. Ophthalmic Physiol Opt 2014; 34:502-8.

11. Ferreira SRS, Glasenapp R, Flores R, organizadores. Tuberculose na atenção primaria à saúde. Brasília: Porto Alegre: Ministério da Saúde/Hospital Nossa Senhora da Conceição; 2011.

12. Victora CG, Barreto ML, do Carmo Leal M, Monteiro CA, Schmidt MI, Paim J, et al. Health conditions and health-policy innovations in Brazil: the way forward. Lancet 2011; 377:2042-53.

13. Thery H, Mello-Thery NA. Disparidades e dinâmicas territoriais no Brasil. Revista do Departamento de Geografia 2012; Volume Especial 30 anos:68-91.

14. Pelissari DM, Rocha MS, Bartholomay P, Sanchez MN, Duarte EC, Arakaki-Sanchez D, et al. Identifying socioeconomic, epidemiological and operational scenarios for tuberculosis control in Brazil: an ecological study. BMJ Open 2018; 8:e018545. 
15. Bodstein R. Atenção básica na agenda da saúde. Ciênc Saúde Coletiva 2002; 7:401-12.

16. Escorel S, Giovanella L, Mendonça MHM, Senna MCM. O Programa de Saúde da Família e a construção de um novo modelo para a atenção básica no Brasil. Rev Panam Salud Pública 2007; 21:164-76.

17. Ministério da Saúde. Portaria no 3.925, de 13 de novembro de 1998. Diário Oficial da União 1998; 17 nov.

18. Ministério da Saúde. Portaria no 373, de 27 de fevereiro de 2002. Norma Operacional da Assistência à Saúde - NOAS-SUS 01/2002. Diário Oficial da União 2002; 28 fev.

19. Ministério da Saúde. Portaria no 399, de 22 de fevereiro de 2006. Divulga o Pacto pela Saúde 2006 - Consolidação do SUS e aprova as Diretrizes Operacionais do Referido Pacto. Diário Oficial da União 2006; 23 fev.

20. Secretaria de Vigilância em Saúde, Ministério da Saúde. Detectar, tratar e curar: desafios e estratégias brasileiras frente à tuberculose. Boletim Epidemiológico 2015; 46(9).

21. Trindade LL, Pires DEP. Implicações dos modelos assistenciais da atenção básica nas cargas de trabalho dos profissionais de saúde. Texto Contexto Enferm 2013; 22:36-42.
22. Machado CV, Lima LD, Viana LS. Configuração da Atenção Básica e do Programa Saúde da Família em grandes municípios do Rio de Janeiro, Brasil. Cad Saúde Pública 2008; 24 Suppl 1:S42-57.

23. Caetano R, Dain S. O Programa de Saúde da Família e a reestruturação da atenção básica à saúde nos grandes centros urbanos: velhos problemas, novos desafios. Physis (Rio J.) 2002; 12:11-21.

24. Monroe AA, Gonzales RIC, Palha PF, Sassaki CM, Ruffino Netto A, Vendramini SHF, et al. Envolvimento de equipes da atenção básica à saúde no controle da tuberculose. Rev Esc Enferm USP 2008; 42:262-7.

25. Macinko J, Starfield B, Shi L. The contribution of primary care systems to health outcomes within Organization for Economic Cooperation and Development (OECD) countries, 1970-1998. Health Serv Res 2003; 38:831-65.

26. Pelissari DM, Bartholomay P, Jacobs MG, Arakaki-Sanchez D, Anjos DSO, Costa MLS, et al. Offer of primary care services and detection of tuberculosis incidence in Brazil. Rev Saúde Pública 2017; 52:53. 


\section{Abstract}

This study aimed to validate a method for classification of healthcare services in Brazil (basic care vs. other levels) and describe the decentralization of tuberculosis (TB) care to basic services (2002 to 2016). The healthcare services that reported and followed TB cases were classified as either "basic care" or "other levels" based on the type of establishment registered in the Brazilian National Registry of Healthcare Establishments (CNES, in Portuguese). The study estimated the agreement between this classification with a previous classification performed in 2013 by Brazil's state and local tuberculosis programs. Using the CNES registry, the authors then calculated the percentage of $T B$ patients treated in basic care from 2002 to 2016. Agreement was 94.4\%, and overall kappa index was 0.86 . There was a relative increment of $31.2 \%$ in TB care provided by basic services (from 50.9\% in 2002 to 66.8\% in 2016). All regions of Brazil showed an increase in this percentage, except the South. The classification based on the CNES registry allowed analyzing the trend in decentralization of TB treatment to basic healthcare services in Brazil.

Tuberculosis; Primary Health Care; Health Information Systems; Decentralization

\section{Resumen}

Los objetivos de este artículo fueron validar un método de clasificación de los servicios de salud (atención básica vs. otros niveles) y describir la descentralización del cuidado de la tuberculosis $(T B)$ hacia los servicios de atención básica en Brasil, durante el período de 2002 a 2016. Los servicios de salud que notificaron y efectuaron el seguimiento a personas con TB fueron clasificados como "atención básica" u "otros niveles", considerándose el tipo de establecimiento registrado en el Registro Nacional de Establecimientos de Salud (CNES). Se estimó la concordancia entre esta clasificación y una realizada en 2013 por los programas estatales y municipales de TB. Posteriormente, utilizando el CNES, se calculó el porcentaje de personas con TB atendidas en la atención básica durante el período de 2002 a 2016. La concordancia fue de 94,4\% y el indice kappa global fue 0,86. Hubo un incremento relativo de un $31,2 \%$ del manejo de la TB en la atención básica (de un 50,9\% en 2002 hasta un 66, 8\% en 2016). Todas las regiones presentaron un aumento de ese porcentaje, excepto la región Sur. La clasificación, basada en el CNES, permitió analizar la evolución de la descentralización del manejo de la TB hacia la atención básica en Brasil.

Tuberculosis; Atención Primaria de Salud; Sistemas de Información en Salud; Descentralización
Recebido em 03/Out/2017

Versão final reapresentada em 02/Jul/2018

Aprovado em 21/Set/2018 